\title{
Balanced Network Flows. IV. Duality and Structure Theory
}

\author{
Christian Fremuth-Paeger, Dieter Jungnickel \\ Lehrstuhl für Diskrete Mathematik, Optimierung und Operations Research, University of Augsburg, \\ D-86135 Augsburg, Germany
}

\begin{abstract}
In this paper, we present a theory for generalized graph factor problems which is basically equivalent to Chapter 10 in Lovasz/Plummer [8] but considerably more intuitive since it uses the language of balanced network flows. The highlights are extensions of the Gallai-Edmonds decomposition and an intuitive development of Lovasz's factor theorem. (c) 2001 John Wiley \& Sons, Inc.
\end{abstract}

Keywords: capacitated matching problems; network flows; factor theorems; Gallai-Edmonds decomposition; odd sets; barriers; cuts; duality

\section{Preliminaries}

The present paper continues our study of balanced network flows and general matching problems [2, 3, 4]. Problems of finding maximum matchings, minimumdeficiency matchings, and even factors of graphs have dual formulations by so-called odd cuts. Minimummaximum results have been derived for various matching problems. However, statements in terms of generalized matching problems are far less intuitive than equivalent results in terms of the balanced network flow approach.

In [2], we stated problems, reduction mechanisms, and a purely primal maximality result. We do not repeat the definitions, but refer to Sections 2, 3, 4, 5, and 9 of that paper. In particular, the cut $Q(N, f):=Q(N(f))$, which separates the strictly $s$-reachable nodes in the residual network $N(f)$, appears as an optimum dual solution and plays the central role in what follows.

Received May 1998; accepted December 2000

Correspondence to: C. Fremuth-Paeger; (e-mail: christian.fremuth@ math.uni-augsburg.de)

(c) 2001 John Wiley \& Sons, Inc.

\section{EDGE CUTS AND DUALITY}

Let $N$ be a balanced flow network, and $f$ a balanced $s t$-flow on $N$ so that no valid st-path in $N(f)$ exists. In this section, we show that $f$ is maximum balanced by a convenient duality theorem. The proof is independent of Theorem 4.1 in [2] (Balanced Flow Decomposition). Again, Theorem 4.2 (Augmenting Path Theorem) can be obtained as a corollary. First, we determine the flow value of $f$ :

For this goal, let $d$ denote the balanced distance labels in $N(f)$, and choose nodes $u, v \in V(N)$ with $d(u)<$ $\infty, d(v)=\infty$ and rescap $(u, v)>0$. We have $d\left(u^{\prime}\right)<\infty$ and $\operatorname{rescap}(u v)=1$ since every valid $s u$-path must traverse $v^{\prime} u^{\prime}$ (otherwise, $v$ would be reachable). Hence, $u$ is in a nucleus $U$ of $N(f)$. We know by Theorem 9.13 in [2] (Base Identity) that $v^{\prime} u^{\prime}$ is the prop of this nucleus and that $\operatorname{rescap}(U, T)=1$. We denote the number of nuclei by $k$ and observe that

$$
\operatorname{rescap}(S, T)=\operatorname{rescap}(\mathscr{C}, T)=k \text {. }
$$

If we translate our observations from $N(f)$ to the flow network $N$, we have

Theorem 23.1. Let $N$ be a balanced flow network, and $f$ a balanced flow on $N$ so that there is no valid st-path in $N(f)$. Then, $\operatorname{val}(f)=\operatorname{cap}(Q(N, f))-k$ holds.

Proof: Let $f$ be an arbitrary st-flow, and $[S, T]$ an arbitrary st-cut on a flow network $N$. If we denote by $e(v):=\sum_{u v \in A(N)} f(u v)-\sum_{v u \in A(N)} f(v u)$ the flow excess at the node $v$, we have

$$
\begin{aligned}
\operatorname{val}(f) & =e(s)=\sum_{v \in S} e(v) \\
& =\sum_{u \in S}\left(\sum_{u v \in A(N)} f(u v)-\sum_{v u \in A(N)} f(v u)\right) \\
& =f(S, T)-f(T, S) .
\end{aligned}
$$


For given subsets $X \subseteq S, Y \subseteq T$, we observe that

$$
\begin{aligned}
\operatorname{rescap}(X, Y) & =\sum_{\substack{u \in X \\
v \in Y}} \operatorname{rescap}(u, v) \\
& =\sum_{\substack{u \in X \\
v \in Y}}[f(v, u)+\operatorname{cap}(u, v)-f(u, v)] \\
& =f(Y, X)-f(X, Y)+\operatorname{cap}(X, Y) .
\end{aligned}
$$

Taking $X=S, Y=T$ yields $\operatorname{val}(f)=\operatorname{cap}(S, T)-$ $\operatorname{rescap}(S, T)$.

Here, we also show that the value of a maximum balanced $s t$-flow is restricted by the costs of a minimum $s t$-cut. It is easy to construct a network where the duality gap is strict. Such an example was given in Figure 3 in [2]. It is well known (and easy to see) that an stflow $f$ and an $s t$-cut $Q$ with $\operatorname{val}(f)=\operatorname{cap}(Q)$ exist. In our special situation, we can close the duality gap artificially.

Let $Q=[S, T]$ be an arbitrary st-cut. We put

$$
\begin{aligned}
& \mathscr{A}(Q):=\left\{v \in S: v^{\prime} \in T\right\} \\
& \mathscr{B}(Q):=\left\{v \in T: v^{\prime} \in S\right\} \\
& \mathscr{C}(Q):=\left\{v \in S: v^{\prime} \in S\right\} \\
& \mathscr{D}(Q):=\left\{v \in T: v^{\prime} \in T\right\} .
\end{aligned}
$$

The set $\mathscr{C}(Q)$ is called the core of $Q$, and the connected components of $N[\mathscr{C}(Q)]$ are the nuclei of $Q$. These definitions are obvious extensions of the definitions given in [2]. A nucleus $U$ is called odd iff $\operatorname{cap}(U, T)$ is odd. The total number of odd nuclei is denoted by odd $(Q)$. A minimum balanced $s t$-cut is an $s t$-cut with minimum balanced capacity

$$
\operatorname{balcap}(Q):=\operatorname{cap}(Q)-\operatorname{odd}(Q) .
$$

Before we study cuts in general, we establish the identity $\operatorname{val}(f)=\operatorname{balcap}(Q)$, where $Q=Q(N, f)$, and $f$ cannot be augmented validly.

Lemma 23.2. $\mathscr{C}$ and $\mathscr{D}$ are nonadjacent, that is, $[\mathscr{C}, \mathscr{D}]=[\mathscr{D}, \mathscr{C}]=\varnothing$.

Proof: Assume an arc $v w \in[\mathscr{C}, \mathscr{D}]$, which implies that $w^{\prime} v^{\prime} \in[\mathscr{D}, \mathscr{C}]$. In particular, we have $\operatorname{rescap}(v, w)>0$ or $\operatorname{rescap}\left(v^{\prime}, w^{\prime}\right)>0$. In the former case, every $d(v)$ path can be extended to a valid $s w$-path by $v w$; in the latter, every $d\left(v^{\prime}\right)$-path can be extended to a valid $s w^{\prime}$ path by $v^{\prime} w^{\prime}$. In either case, we obtain a contradiction to the choice of $w$.

Theorem 23.3. Let $N$ be a balanced-flow network, and $f$ a balanced flow on $N$ and assume that t is not s-reachable in $N(f)$. Then, every nucleus is odd.

Proof: For given node sets $M, \tilde{M} \subseteq V(N)$, let

$$
M_{O}:=M \cap \operatorname{Outer}(N), M_{I}:=M \cap \operatorname{Inner}(N)
$$

and

$$
F_{M \tilde{M}}:=f\left(M_{O}, \tilde{M}_{I}\right), G_{M \tilde{M}}:=f\left(M_{I}, \tilde{M}_{O}\right) .
$$

Let $U$ be a nucleus and $\mathscr{B}:=\mathscr{A}^{\prime}$. Recall that nuclei are self-complementary. Note that $\mathscr{B}_{O}^{\prime}=\mathscr{A}_{I}$ and $\mathscr{A}_{O}^{\prime}=\mathscr{B}_{I}$ so that $F_{U \mathscr{A}}=F_{\mathscr{B} U}$ and $G_{\mathscr{A} U}=G_{U \mathscr{B}}$. By Lemma 0, we have

$$
\begin{aligned}
f(U, T) & =F_{U \mathscr{B}}+G_{U \mathscr{B}}=F_{U \mathscr{B}}+G_{\mathscr{A} U}, \\
f(T, U) & =F_{\mathscr{B} U}+G_{\mathscr{B} U}=F_{U \mathscr{A}}+G_{\mathscr{B} U} .
\end{aligned}
$$

Addition of the flow-conservation conditions for the nodes in $U_{O}$ yields

$$
0=F_{U U}+F_{U \mathscr{A}}+F_{U \mathscr{B}}-G_{U U}-G_{\mathscr{A} U}-G_{\mathscr{B} U}
$$

Since $U$ is a nucleus, we have $\operatorname{rescap}(U, T)=1$, and by Eq. (1),

$$
\operatorname{cap}(U, T)=1+f(U, T)-f(T, U) .
$$

If we substitute $f(U, T)$ and $f(T, U)$ by the Eqs. (3) and (4) and add Eq. (5), we see that

$$
\operatorname{cap}(U, T)=1+F_{U U}+2 F_{U \mathscr{B}}-G_{U U}-2 G_{\mathscr{B} U} .
$$

Since $\left[U_{O}, U_{I}\right]$ and $\left[U_{I}, U_{O}\right]$ are self-complementary and $f$ is balanced, $F_{U U}$ and $G_{U U}$ are even. Hence, $\operatorname{cap}(U, T)$ is an odd number.

Theorem 23.4. (Duality Theorem). Let $f$ be a balanced $s t$-flow and $Q$ an $s t$-cut of the balanced flow network $N$. Then,

$$
\operatorname{val}(f) \leq \operatorname{balcap}(Q)
$$

holds. Furthermore, equality holds iff $f$ is a maximum balanced $s t$-flow, and $Q$ is a minimum balanced $s t$-cut.

Proof: Let $f$ be an arbitrary balanced flow, $Q=[S, T]$ an arbitrary $s t$-cut, and $U$ an odd nucleus of $Q$. We use the notation of the last proof but replace $Q(N, f)$ by $Q$, taking $\mathscr{A}:=\mathscr{A}(Q), \mathscr{B}:=\mathscr{A}(Q)^{\prime}, \mathscr{C}:=\mathscr{C}(Q)$, and $\mathscr{D}:=\mathscr{D}(Q)$.

If $U$ and $\mathscr{D}$ are adjacent, we have $\operatorname{rescap}(u, v)>0$ or $\operatorname{rescap}\left(u^{\prime}, v^{\prime}\right)>0$, in particular, $\operatorname{rescap}(U, T) \geq 1$. So suppose, otherwise, that $[U, \mathscr{D}]=\varnothing$. Then, we get the equations (3) and (5) as in the situation of Theorem 0. Addition yields

$$
f(U, T)=F_{U U}+2 F_{U \mathscr{B}}-G_{U U}+F_{U \mathscr{A}}-G_{\mathscr{B} U} .
$$

If we have $f(T, U)=0$, then the right-hand side is even. But $\operatorname{cap}(U, T)$ is odd so that, $\operatorname{cap}(U, T)-f(U, T) \geq 1$. In either case, we have

$$
\operatorname{rescap}(U, T)=\operatorname{cap}(U, T)-f(U, T)+f(T, U) \geq 1 .
$$


Adding this inequality over all nuclei yields $\operatorname{rescap}(S, T)$ $\geq \operatorname{odd}(Q)$, and, hence,

$$
\operatorname{val}(f)=f(S, T)-f(T, S) \leq \operatorname{cap}(S, T)-\operatorname{odd}(Q) .
$$

The strong duality property follows by Theorems 23.1 and 23.3.

This statement was established by Tutte [10] first. Our proof generalizes the approach of Kocay/Stone [6] who introduced a more familiar notation.

\section{CANONICAL DECOMPOSITION}

In $[2,3,4]$, we presented a powerful machinery for solving various matching problems. We spent much effort in the design of BNS algorithms which allow one to augment a given matching in terms of a corresponding balanced-flow network.

Actually, the BNS algorithms not only provide a single solution but give us information about the common structure of all optimum solutions. The crux is the partition of the node set of a balanced-flow network $N$ into $\mathscr{A}(N, f), \mathscr{B}(N, f), \mathscr{C}(N, f)$, and $\mathscr{D}(N, f)$ according to some maximum balanced st-flow $f$.

As it turns out, these sets are independent of the specific flow $f$. This observation will establish a generalization of the well-known Gallai-Edmonds decomposition (see [8]) which helps to describe the common structure of all maximum cardinality matchings of a simple graph.

Lemma 24.1. Let $f$ be a maximum balanced st-flow on the balanced-flow network $N$ and $s w \in A(N)$. Then, $w$ is strictly s-reachable in $N(f)$ iff there is a maximum balanced st-flow $g$ on $N$ such that $g(s w)<c a p(s w)$ holds.

\section{Proof:}

$(\rightarrow)$ If $f(s w)<\operatorname{cap}(s w)$ holds, we just have to put $g: \equiv f$. Assume, otherwise, that $f(s w)$ and $c a p(s w)$ are equal and that $w$ is reachable in $N(f)$. Let $r$ be a valid $s w$-path. Then, $p:=r \circ w s$ is a valid cycle and $g:=f+f_{p}+f_{p^{\prime}}$ is a maximum balanced $s t$-flow on $N$ such that $g(s w)<$ cap $(s w)$ holds.

$(\leftarrow)$ If $f(s w)<c a p(s w)$ holds, then $w$ is strictly $s$ reachable in $N(f)$. Now suppose that $f(s w)=c a p(s w)$ and that another maximum balanced flow $g$ on $N$ with $g(s w)<\operatorname{cap}(s w)$ exists. Then, by Theorem 4.1 in [2], a valid cycle $p$ in $N(f)$ exists which traverses the arc $w s$. In particular, we have $d(w)<\infty$.

The preceding statement concerned only parts of the network, but indicates that reachability is independent of the choice of a particular maximum balanced flow. The general result uses the duality theorem of the last section. We can characterize the canonical $s t$-cut $Q(N)$ in terms of the other minimum balanced cuts:
Theorem 24.2. Let $N$ be a balanced flow network, and $f$ a maximum balanced st-flow on $N$. Then, $v \in V(N)$ is strictly s-reachable with respect to $N(f)$ iff $v \in S$ for every minimum balanced st-cut $[S, T]$ of $N$.

Proof: Since $Q(N, f)$ is minimum balanced by Theorem 23.4, the backward direction is obvious.

On the other hand, let $p$ be a valid $s v$-path in $N(f) ; u$, the predecessor of $v$ on $p$; and $Q=[S, T]$, a minimum balanced st-cut of $N$. Assume that $v \in T$ and, without loss of generality, $u \in S$. By Theorem 23.4, we have

$$
\operatorname{val}(f)=\operatorname{balcap}(Q)=\operatorname{cap}(S, T)-\operatorname{odd}(Q),
$$

that is, $\operatorname{rescap}(S, T)=\operatorname{odd}(Q)$. Hence, equality must hold for all inequalities in the proof of Theorem 23.4 which shows $\operatorname{rescap}(\mathscr{A}, T)=0$. We have $\operatorname{rescap}(u, v)>$ 0 so that $u$ is in a nucleus of $Q$. By Theorem 9.13 in [2] (Base Identity), we obtain rescap $(u v)=1$ and that $p$ traverses $v^{\prime} u^{\prime}$ also, a contradiction.

Corollary 24.3. Let $N$ be a balanced-flow network, and $f$ a maximum balanced st-flow on $N$. The minimum balanced st-cut $Q(N):=Q(N, f)$ is well defined, that is, $Q(N, f)$ is independent of the special choice of $f$.

Proof: Observe that $S(N, f)=\cap\{S:[S, T]$ minimum balanced $s t$-cut $\}$.

Let $\mathcal{M}$ be an instance for the extended matching problem. Recall that $N_{\mathscr{M}}^{\#}$ is the network $N_{\mathscr{M}}^{*}$ where $\operatorname{cap}\left(s^{*}, t\right)=$ $\operatorname{cap}\left(s, t^{*}\right)=a(V(\mathscr{M}))-\operatorname{def}(\mathscr{M})$ is set. In [2], we showed that a maximum balanced $s t$-flow on $N_{\mathcal{M}}^{\#}$ corresponds to a minimum-deficiency matching. The sets

$$
\begin{aligned}
& \mathscr{A}(\mathscr{M}):=\mathscr{A}\left(N_{\mathcal{M}}^{\#}\right) \cap V(\mathscr{M}), \\
& \mathscr{B}(\mathcal{M}):=\mathscr{B}\left(N_{\mathcal{M}}^{\#}\right) \cap V(\mathscr{M}), \\
& \mathscr{C}(\mathcal{M}):=\mathscr{C}\left(N_{M}^{\#}\right) \cap V(\mathscr{M}), \\
& \mathscr{D}(\mathcal{M}):=\mathscr{D}\left(N_{\mathcal{M}}^{\#}\right) \cap V(\mathcal{M})
\end{aligned}
$$

form the canonical decomposition for $\mathcal{M}$. If upper and lower-degree constraints coincide, one can exchange in this definition $N_{\mathcal{M}}^{\#}$ by the network $N_{\mathcal{M}}$, which is almost the same. Note that any matching algorithm which depends on augmentation and a balanced network search will finally compute the canonical decomposition.

As an example, consider the graph $G$ of Figure 1 and its subgraphs with maximum node degree 3. Obviously, a 3-factor cannot exist since the number of nodes is odd, and the matching $x$ that consists of the boldface edges is maximum. The canonical decomposition is

$$
\begin{aligned}
& \mathscr{A}(\mathscr{M})=\{1,2,3,5,6,9,11,12\} \\
& \mathscr{B}(\mathscr{M})=\{4,7,8\} \\
& \mathscr{C}(\mathscr{M})=\{13,14,15\} \\
& \mathscr{D}(\mathscr{M})=\{10\} .
\end{aligned}
$$




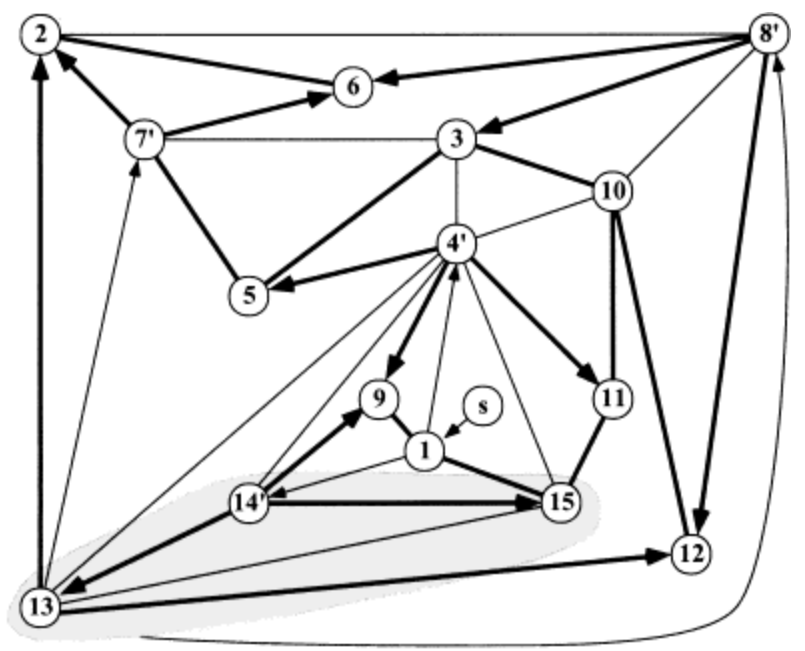

FIG. 1. An example of a canonical decomposition.

The reader is asked to verify the sets in terms of some other maximum matching. If we are concerned with the cardinality matching problem, we write $\mathscr{A}(G), \mathscr{B}(G)$, $\mathscr{C}(G)$, and $\mathscr{D}(G)$. Here, the canonical decomposition is known as the Gallai-Edmonds decomposition. Since this problem is purely combinatorial, we have some more explicit statements. One of the most important is the Gallai-Edmonds Structure Theorem. But this theorem can also be formulated for the $b$-matching problem:

Lemma 24.4. Let $M$ be an instance for the b-matching problem. Then, $\mathscr{A}(\mathcal{M}), \mathscr{C}(\mathcal{M})$, and $\mathscr{D}(\mathcal{M})$ are pairwise nonadjacent and $\mathscr{A}(\mathscr{M})$ is an independent node set.

Proof: Consider $Q\left(N_{\mu}\right)$, which is a minimum balanced st-cut. Note that balcap $(Q) \leq \operatorname{balcap}(s, V(M))=b(V)$. Since we have $\operatorname{cap}\left(u, v^{\prime}\right)>b(V)$ for every edge $\{u, v\} \in$ $E(\mathcal{M}), u v^{\prime}$ cannot be an arc of the cut $Q$. But this means that $\left[\mathscr{A}, \mathscr{A}^{\prime}\right]=\left[\mathscr{C}, \mathscr{A}^{\prime}\right]=\left[\mathscr{A}, \mathscr{D}^{\prime}\right]=\varnothing$ and $\left[\mathscr{C}, \mathscr{D}^{\prime}\right]=\varnothing$ holds by Lemma 23.2.

Theorem 24.5. Let $\mathcal{M}$ be an instance for the b-matching problem, $b:=b(\mathcal{M})$ and $G:=G(\mathcal{M})$. Then, there is a partition $V(\mathscr{M})=\mathscr{A} \uplus \mathscr{B} \uplus \mathscr{C} \uplus \mathscr{D}$ so that the following statements hold:

(a) The connected components of $G[\mathscr{D}]$ admit perfect bmatchings.

(b) The connected components of $G[\mathscr{C}]$ are factor-critical.

(c) Every maximum b-assignment of $G[\mathscr{B}, \mathscr{A} \uplus \mathscr{C}]$ saturates $\mathscr{B}$.

(d) Every maximum b-matching of $G$ splits into a perfect $b$-matching of $G[\mathscr{D}]$, near-perfect b-matchings of the connected components of $G[\mathscr{C}]$, and a complete $b$ assignment of $G[\mathscr{B}, \mathscr{A} \uplus \mathscr{C}]$.

Proof: Let $f$ be a maximum balanced flow on $N_{\mu}$, and $x$, the corresponding matching of $G$. We choose $\mathscr{A}=$ $\mathscr{A}(\mathcal{M}), \mathscr{B}=\mathscr{B}(\mathcal{M}), \mathscr{C}=\mathscr{C}(\mathcal{M})$, and $\mathscr{D}=\mathscr{D}(\mathcal{M})$.
Since every node of $\mathscr{B}^{\prime}$ but no node of $\mathscr{D}$ is strictly $s$-reachable, we have $f\left(\mathscr{D}, \mathscr{B}^{\prime}\right)=0$. By the preceding lemma, the nodes in $\mathscr{D}$ are matched among themselves, and $x[\mathscr{D}]$ is a perfect $b$-matching of $\mathscr{D}$. Assertion (a) follows.

Let $U$ be a nucleus, $u:=\operatorname{base}(U)$, and observe that $b(u)>0$. If rescap $(s u)=0$ holds, we exchange $f$ by the maximum balanced flow $f+f_{r}+f_{r^{\prime}}$, where $r:=$ $p \circ u s$ holds for some valid su-path $p$. This does not affect the flow values on $U$. After this operation, we have $\operatorname{rescap}(s u)=1$. If $\{v, w\}$ is an edge of $G$ with $v \in U$, $W \notin U$, then we have $w \in B$ and $f\left(w v^{\prime}\right)=0$, since, otherwise, $w$ would be reachable.

Hence, the nodes $v \in U_{O}:=U \cap V(\mathcal{M}), v \neq u$ are matched to other nodes of $U_{O}$ only, and, hence, $x\left[U_{O}\right]$ is a near-perfect $b$-matching which is $u$-deficient. Let $v \in U_{O}, v \neq u$, and $r:=p \circ v s$ for some valid $s v$-path $p$ in $N_{\mu}(f)$. Let $x_{v}$ be the maximum $b$-matching corresponding to $f+f_{r}+f_{r^{\prime}}$. Then, $x_{v}\left[U_{O}\right]$ is $v$-deficient and $U_{O}$ is factor-critical. This is assertion (b).

By Corollary 24.3 , the nodes of $\mathscr{B}$ are saturated by every maximum matching of $G$. Note that we have $f\left(\mathscr{B}, \mathscr{B}^{\prime}\right)=f\left(\mathscr{B}, \mathscr{D}^{\prime}\right)=0$, which gives (c). Assertion (d) follows directly by the above discussion.

Corollary 24.6. (Gallai-Edmonds Structure Theorem). Let $G$ be a simple graph. Then, there is a partition $V(G)=\mathscr{A} \biguplus \mathscr{B} \biguplus \mathscr{C} \biguplus \mathscr{D}$ so that the following statements hold:

(a) The connected components of $G[\mathscr{D}]$ admit 1-factors.

(b) The connected components of $G[\mathscr{C}]$ are factor-critical.

(c) Every maximum assignment of $G[\mathscr{B}, \mathscr{A} \uplus \mathscr{C}]$ covers $\mathscr{B}$.

(d) Every maximum matching of $G$ splits into a 1-factor of $G[\mathscr{D}]$, near-perfect matchings of the connected components of $G[\mathscr{C}]$, and a complete assignment of $G[\mathscr{B}, \mathscr{A} \uplus \mathscr{C}]$.

It is obvious now that one can define the canonical decomposition in terms of the underlying matching problem. This is also possible in the general situation. To see this, we extend the definitions given in [2], letting

$$
\operatorname{def}(x):=\sum_{v \in V(\mathscr{M})} \max \left\{0, a(v)-\operatorname{deg}_{x}(v), \operatorname{deg}_{x}(v)-b(v)\right\}
$$

be the deficiency of an arbitrary subgraph $x$ of the multigraph $G(M)$. A node $v$ with $b(v)<d e g_{x}(v)$ is called supersaturated. A subgraph $x$ which has minimum deficiency among all subgraphs is called optimal.

Theorem 24.7. There always is an optimal matching.

Proof: Let $x$ be an optimal subgraph. If $x$ is a matching, we are finished. So assume that $\operatorname{deg}_{x}(v)>b(v)$ for some node $v \in V(\mathcal{M})$, $e$, an arc incident with $v$, and $w$, the other end node of $e$. 
Denote by $y$ the subgraph which is obtained from $x$ by omitting $e$. If $w=v$, then we have $\operatorname{deg}_{x}(v)=b(v)+1$ and $a(v)=b(v)$, since, otherwise, $\operatorname{de} f(y)<\operatorname{def}(x)$ would result. If $w \neq v$, then $\operatorname{deg}_{x}(w) \leq a(w)$ holds for the same reason. In either case, $y$ is optimal. If we proceed with such update steps, we finally reach an optimal matching.

Corollary 24.8. A given node is deficient in some minimum deficiency matching iff it is deficient in some optimal subgraph.

\section{Theorem 24.9.}

$V^{-}(\mathcal{M}):=\{v \in V(\mathcal{M}):$

There are $v$-deficient optimal subgraphs $\}=\mathscr{A} \biguplus \mathscr{C}$.

Proof: Let $v \in V(\mathscr{M})$. If $a(v)>0$, then apply Corollary 24.8, Lemma 24.1, and Lemma 5.2 of [2] to see that $v \in V^{-}(\mathscr{M})$ iff $v \in \mathscr{A} \biguplus \mathscr{C}$.

If $a(v)=0$, then no subgraph is $v$-deficient. Suppose that $f$ is a maximum balanced $s^{*} t^{*}$-flow on $N_{M}^{\#}$ and assume the existence of a valid $s^{*} v$-path in $N_{M}^{\#}(f)$. Since $s$ cannot be traversed, $v$ must be reached by an arc $u^{\prime} v$, $u \in V(\mathcal{M})$. But, then, $f\left(v, u^{\prime}\right)>0$ and $f(s, v)>0$ follow. So at least one of $s$ and $t$ would be strictly $s$-reachable, a contradiction.

\section{Theorem 24.10.}

$V^{+}(\mathcal{M}):=\{v \in V(\mathcal{M}):$

There are $v$-supersaturated optimal subgraphs $\}=\mathscr{B} \uplus \mathscr{C}$.

Proof:

$(\subseteq)$ Let a $v$-supersaturated optimal subgraph be given. This subgraph can be turned into an optimal matching as described in the proof of Theorem 24.7. Even more, we may assume that $v$ is still supersaturated before the last deletion step.

Let $x$ be the optimal subgraph just before this ultimate step, $e$ be the edge deleted and $y$ be the final matching. We have $\operatorname{deg}_{x}(v)=b(v)+1$ and $\operatorname{deg}_{x}(w) \leq b(w)$ for $w \in V(\mathcal{M})-v$. Suppose that $y$ corresponds to the flow $f$ on $N_{\mu}^{\#}$. If $w$ is the end node of $e$ other than $v$, then $y$ is $w$-deficient. Hence, $\left(s^{*}, w, v^{\prime}\right)$ is a valid $s^{*} v^{\prime}$-path in $N_{M}^{\#}(f)$.

() Let $f$ be a maximum balanced $s^{*} t^{*}$-flow on $N_{\mu}^{\#}, x$ be the corresponding matching, and $v \in V(\mathscr{M})$ so that a valid $s^{*} v^{\prime}$-path $p$ in $N_{\mathcal{M}}^{\#}(f)$ exists. Since neither $s$ nor $t$ is strictly reachable, we have $\operatorname{rescap}\left(v^{\prime}, t\right)=0$. Since $f$ is maximum balanced, we have $\operatorname{rescap}\left(v^{\prime}, t^{*}\right) \leq 1$. If $\operatorname{rescap}\left(v^{\prime}, t^{*}\right)=1$, then $p$ must start with the arc $s^{*} v$.

If we augment $f$ along $p \circ\left(v^{\prime}, t, s, t^{*}\right)$ and the complementary path, the resulting flow $g$ violates the bounds $\operatorname{cap}\left(v^{\prime}, t\right)$ and $\operatorname{cap}\left(t, t^{*}\right)$. If we replace in $\mathcal{M}$ the bound $b(v)$ by $\tilde{b}(v):=b(v)+1$, then $g$ is an admissible balanced flow on $N_{\mu}$ again [this requires us to verify $\tilde{b}(v) \leq \operatorname{deg}(v)]$.

Note that we have $g\left(v^{\prime}, t^{*}\right)=a(v)$ and $g\left(v^{\prime}, t\right)=$ $b(v)-a(v)+1$. Actually, $g$ corresponds to a matching $y$ in the modified problem. In the original problem, $y$ is $v$-supersaturated. If $p$ starts with the $\operatorname{arc} s w$, then $x$ is $w$-deficient and $\operatorname{deg}_{y}(w)=\operatorname{deg}_{x}(w)+1$. Hence, $y$ is even optimal.

We obtain $\mathscr{A}=V^{+}(\mathscr{M}) \backslash V^{-}(\mathcal{M}), \mathscr{B}=V^{-}(\mathcal{M}) \backslash V^{+}(\mathscr{M})$, $\mathscr{C}=V^{-}(\mathscr{M}) \cap V^{+}(\mathscr{M})$, and $\mathscr{D}=V(\mathscr{M}) \backslash\left(V^{-}(\mathcal{M}) \cup V^{+}(\mathcal{M})\right)$. For sake of completeness, we mention another construction of the canonical decomposition:

The complementary subgraph network $\bar{M}$ results from $\mathcal{M}$ where the degree bounds $a$ and $b$ are replaced by $\bar{a}: \equiv \operatorname{deg}-b$ and $\bar{b}: \equiv \operatorname{deg}-a$. To a given subgraph $x$, the complementary subgraph is $\bar{x}: \equiv c-x$.

Note that $x$ is $v$-deficient in $M$ iff $\bar{x}$ is $v$-supersaturated in $\bar{M}$. Hence, we get $V^{+}(\bar{M})=V^{-}(\mathscr{M})$ and $V^{-}(\bar{M})=$ $V^{+}(\mathscr{M})$. If we translate the situation to the balanced-flow networks $N_{\mathscr{M}}^{\#}$ (respectively, $N_{\bar{M}}^{\#}$ ), we have $\overline{\mathscr{A}}=\mathscr{B}, \overline{\mathscr{B}}=$ $\mathscr{A}, \overline{\mathscr{C}}=\mathscr{C}$, and $\overline{\mathscr{D}}=\mathscr{D}$. If follows that the canonical decomposition can be constructed from the sets $V^{-}(\mathcal{M})$ and $V^{-}(\bar{M})$ which refer to matchings only.

\section{ODD SETS, BARRIERS, AND FACTOR THEOREMS}

We now present some duality theorems which relate matchings to their dual structures, called barriers. In particular, we give a defect form of Tutte's 1-factor theorem [9] as well as a proof of Lovasz's factor theorem [7] which is the most general duality statement in terms of a graph matching problem.

Let $G$ be a simple graph and $W \subseteq V(G)$. By $\operatorname{odd}(W)$, we denote the number of components with odd cardinality in $G[W]$. Observe $\operatorname{odd}(Q)=\operatorname{odd}(\mathscr{C})$, where $Q$ is the canonical st-cut and $\mathscr{C}$ is the core of the network $N_{G}$. Odd sets give a very intuitive condition for the existence of 1-factors.

Our proof applies the Duality Theorem 23.4 to the network $N_{G}$. Kocay and Stone [6] proved the more general $f$-factor theorem in the same way. A more direct proof of the 1-factor theorem which depends on Hall's Marriage Theorem can be found, for example, in Jungnickel [5].

Theorem 25.1. (1-Factor Theorem).

Let $G$ be a simple graph. Then, there is a matching of $G$ 
with deficiency at most $k$ iff

$$
|X|+k \geq \operatorname{odd}(Y)
$$

holds for arbitrary partitions $V(G)=X \biguplus Y$.

\section{Proof:}

$(\rightarrow)$ If $U$ is an odd component of $G[Y]$, then at least one node in $U$ must be either matched to a node not in $Y$ or unmatched. The assertion follows.

$(\leftarrow)$ Let $f$ be a maximum balanced $s t$-flow on $N_{G}$ again, and $Q:=Q\left(N_{G}\right)$ canonical. Suppose there is no matching with deficiency $k$ or less, that is, $\operatorname{balcap}(Q)=\operatorname{val}(f)<$ $|V(G)|-k$. We can compute balcap $(Q)$ in terms of the canonical decomposition $\mathscr{A}=\mathscr{A}(G), \mathscr{B}=\mathscr{B}(G), \mathscr{C}=$ $\mathscr{C}(G)$, and $\mathscr{D}=\mathscr{D}(G)$. If $Q$ would be the canonical st-cut on $N_{\mathscr{M}}$ where $\mathcal{M}$ is arbitrary, we would have

$$
\begin{aligned}
\operatorname{balcap}(Q)= & \operatorname{cap}\left(\mathscr{A}, \mathscr{A}^{\prime}\right)+\operatorname{cap}\left(\mathscr{A}, \mathscr{D}^{\prime}\right)+\operatorname{cap}\left(\mathscr{C}, \mathscr{A}^{\prime}\right) \\
& +\operatorname{cap}\left(\mathscr{B}^{\prime}, t\right)+\operatorname{cap}\left(\mathscr{C}^{\prime}, t\right) \\
& +\operatorname{cap}(s, \mathscr{B})+\operatorname{cap}(s, \mathscr{D})-\operatorname{odd}(\mathscr{C}) .
\end{aligned}
$$

By Lemma $24.4, \operatorname{cap}\left(\mathscr{A}, \mathscr{A}^{\prime}\right)=\operatorname{cap}\left(\mathscr{A}, \mathscr{D}^{\prime}\right)=$ $\operatorname{cap}\left(\mathscr{C}, \mathscr{A}^{\prime}\right)=0$ holds in our situation. Since we have $\operatorname{cap}(s, W)=|W|$ for every $W \subseteq V(\mathcal{M})$, one obtains

$$
\operatorname{balcap}(Q)=2|\mathscr{B}|+|\mathscr{C}|+|\mathscr{D}|-\operatorname{odd}(\mathscr{C}) .
$$

If we replace $|V(G)|$ by $|\mathscr{A}|+|\mathscr{B}|+|\mathscr{C}|+|\mathscr{D}|$, we have the inequality

$$
|\mathscr{B}|+k<|\mathscr{A}|+\operatorname{odd}(\mathscr{C}) .
$$

Since $\mathscr{D}$ has a 1 -factor by Theorem 24.6 , it has no odd components. On the other hand, $\mathscr{A}$ is an independent node set, and every element, an odd component of $G[\mathscr{A}]$. Since $\mathscr{A}, \mathscr{C}$, and $\mathscr{D}$ are nonadjacent, we have odd $\mathscr{A} \biguplus$ $\mathscr{C} \biguplus \mathscr{D})=\operatorname{odd}(\mathscr{C})+|\mathscr{A}|$. Put $Y:=\mathscr{A} \uplus \mathscr{C} \uplus \mathscr{D}$ and $X:=\mathscr{B}$ to obtain a contradiction.

Actually, this is not the original 1-Factor Theorem of Tutte but Berge's defect form which is a simple extension:

Corollary 25.2. (Berge Formula).

The minimum deficiency of a matching is given by

$$
\max \{\operatorname{odd}(Y)-|X|: V(G)=X \biguplus Y\} .
$$

Similar results can also be obtained for all the capacitated matching problems (see [9, 7]). Unfortunately, the statements are just as difficult to read as are the corresponding proofs. This may indicate that the network flow formulation is more adequate for capacitated matching problems.

The set system $(X, Y)$ which gives equality in (11) is usually called a barrier. To generalize the result, one effectively splits the set $Y$ into two sets:

Let $X, Y$, and $Z$ be disjoint. Then, $Z$ is called $(X, Y)$ odd or simply odd iff $a(v)=b(v)$ for every $v \in Z$ and
$\tau(Z):=b(Z)+c(Y, Z)$ is odd. Otherwise, $Z$ is called even. Unfortunately, this definition is asymmetric. The number of odd-connected components of $G[V \backslash(X \cup Y)]$ is denoted by odd $(X, Y)$.

In the context of the $b$-matching problem and $Y=$ $\mathscr{A}(\mathscr{M}), X=\mathscr{B}(\mathcal{M})$, we have $\tau(Z)=b(Z)$ by Lemma 24.4. In the cardinality matching context, we even have $\tau(Z)=|Z|$. We now investigate the canonical barrier $X=\mathscr{B}(\mathscr{M}), Y=\mathscr{A}(\mathcal{M}), Z=\mathscr{C}(\mathcal{M}) \cup \mathscr{D}(\mathcal{M})$ in the general setting.

Theorem 25.3. Let $X:=\mathscr{B}$ and $Y:=\mathscr{A}, Z:=\mathscr{C} \cup \mathscr{D}$ with $\mathscr{A}, \mathscr{B}, \mathscr{C}, \mathscr{D}$ the canonical decomposition of $\mathcal{M}$.

(a) Every connected component of $G[\mathscr{C}]$ is odd.

(b) Every connected component of $G[\mathscr{D}]$ is even.

(c) $\mathscr{C}$ and $\mathscr{D}$ are nonadjacent.

(d) $\operatorname{odd}(X, Y)=\operatorname{odd}(Q)$, where $Q=Q\left(N_{M}^{\#}\right)$.

(e) Edges joining $\mathscr{A}$ and $\mathscr{D}$ are saturated in any minimum- deficiency matching.

(f) Edges joining $\mathscr{B}$ and $\mathscr{D}$ are void in any minimumdeficiency matching.

\section{Proof:}

(a) Let $\tilde{U}$ be a connected component of $G[\mathscr{C}]$. Then, $U:=\tilde{U} \cup \tilde{U}^{\prime}$ is a nucleus of $N_{\mu}^{\#}$. Neither $s$ nor $t$ is strictly $s^{*}$-reachable, so we have $\operatorname{rescap}(v, s)=\operatorname{rescap}\left(v^{\prime}, t\right)=$ 0 , and, hence, $\operatorname{cap}(s, v)=b(v)-a(v)=0$ for every node $v \in \mathscr{C}$. Moreover, Theorem 23.3 shows that

$$
\begin{aligned}
\operatorname{cap}(U, T)=\operatorname{cap}\left(\tilde{U}, \mathscr{A}^{\prime}\right) & +\operatorname{cap}\left(\tilde{U}^{\prime}, t^{*}\right) \\
= & c(\tilde{U}, \mathscr{A})+b(\tilde{U})=\tau(\tilde{U})
\end{aligned}
$$

is odd.

(b) Let $\tilde{W}$ be a connected component of $G[\mathscr{D}]$, $W:=\tilde{W} \cup \tilde{W}^{\prime}$, and assume that $a(v)=b(v)$ for every $v \in \tilde{W}$. Then,

$$
\begin{aligned}
\operatorname{cap}(S, W)=\operatorname{cap}\left(\mathscr{A}, \tilde{W}^{\prime}\right)+\operatorname{cap}\left(s^{*}, \tilde{W}\right) & \\
& =c(\tilde{W}, \mathscr{A})+b(\tilde{W})=\tau(\tilde{W}) .
\end{aligned}
$$

Since the nodes in $\mathscr{A}$ are strictly $s^{*}$-reachable, but the nodes in $W$ are not, we have $\operatorname{rescap}\left(s^{*}, \tilde{W}\right)=$ $\operatorname{rescap}\left(\mathscr{A}, \tilde{W}^{\prime}\right)=0$. For an arbitrary maximum balanced flow $f$, this can be written as

$$
\begin{aligned}
\operatorname{cap}(S, W)=f(S, W) & =F_{\mathscr{A} W}+G_{\mathscr{A} W} \\
& =F_{\mathscr{A} W}+G_{W \mathscr{B}}, \\
0=f(W, S) & =F_{W \mathscr{A}}+G_{W \mathscr{A}} \\
& =F_{\mathscr{B} W}+G_{W \mathscr{A}},
\end{aligned}
$$

where we use the shorthand notations of the proof of Theorem 23.3 and the sets $\mathscr{A}$ and $\mathscr{B}$ refer to the canonical $s^{*} t^{*}$-cut again. Adding the mass-balance equations for the nodes in $\tilde{W}^{\prime}$ yields

$$
0=G_{W W}+G_{W \mathscr{A}}+G_{W \mathscr{B}}-F_{W W}-F_{\mathscr{A} W}-F_{\mathscr{B} W},
$$


so that

$\operatorname{cap}(S, W)=G_{W W}+G_{W \mathscr{A}}+2 G_{W \mathscr{B}}-F_{W W}-F_{\mathscr{B} W}$.

Adding (15), we see that $\operatorname{cap}(S, W)=G_{W W}+2 G_{W \mathscr{B}}-$ $F_{W W}-2 F_{\mathscr{B} W}$ is even.

(c) This is essentially Lemma 23.2.

(d) This follows directly from assertions (a)-(c), and Theorem 23.3.

(e) Consider the maximum balanced flow $f$ on $N_{\mu}^{\#}$ corresponding to some minimum-deficiency matching. As in the proof of assertion (b), we obtain $f\left(\mathscr{A}, \mathscr{D}^{\prime}\right)=$ $\operatorname{cap}\left(\mathscr{A}, \mathscr{D}^{\prime}\right)$.

(f) Similar to assertion (e).

One can also prove Lovasz's factor theorem, and even its defect form, with the approach given in [6]. To do this, one applies Theorem 23.4 to the network $N_{\mu}^{\#}$ instead of $N_{\mu}$ :

\section{Theorem 25.4. (Factor Theorem).}

Let $\mathcal{M}$ be a subgraph network. Then, there is a subgraph of $G$ with deficiency $k$ or less iff

$$
b(X)+c(Y, Y \cup Z)+k \geq \operatorname{odd}(X, Y)+a(Y)
$$

holds for arbitrary partitions $V(G)=X \uplus Y \uplus Z$. This inequality can also be written as

$$
b(X)+\bar{b}(Y)+k \geq \operatorname{odd}(X, Y)+c(X, Y) .
$$

Proof: We define an $s^{*} t^{*}$-cut $Q=[S, T]$ by taking $S:=\mathscr{A} \cup \mathscr{B}^{\prime} \cup \mathscr{C} \cup \mathscr{C}^{\prime} \cup\left\{s^{*}\right\}$ and $T:=\mathscr{B} \cup \mathscr{A}^{\prime} \cup$ $\mathscr{D} \cup \mathscr{D}^{\prime} \cup\left\{s, t, t^{*}\right\}$, where $\mathscr{A}:=Y, \mathscr{B}:=X$ and

$$
\begin{aligned}
& \mathscr{C}:=\cup\{U: U \text { is an odd component of } Z\} \\
& \mathscr{D}:=\cup\{U: U \text { is an even component of } Z\} .
\end{aligned}
$$

By choice of $\mathscr{C}$, we have $\operatorname{odd}(Q)=\operatorname{odd}(Z), \operatorname{cap}\left(\mathscr{C}, \mathscr{D}^{\prime}\right)=$ 0 , and $\operatorname{cap}\left(\mathscr{C}^{\prime}, t\right)=0$ since $a(\mathscr{C})=b(\mathscr{C})$. We obtain

$$
\begin{aligned}
\operatorname{balcap}(Q)= & \operatorname{cap}\left(\mathscr{A}, \mathscr{A}^{\prime}\right)+\operatorname{cap}\left(\mathscr{A}, \mathscr{D}^{\prime}\right)+\operatorname{cap}\left(\mathscr{C}, \mathscr{A}^{\prime}\right) \\
& +\operatorname{cap}\left(\mathscr{B}^{\prime}, t^{*}\right)+\operatorname{cap}\left(\mathscr{B}^{\prime}, t\right)+\operatorname{cap}\left(\mathscr{C}^{\prime}, t^{*}\right) \\
& +\operatorname{cap}\left(s^{*}, \mathscr{B}\right)+\operatorname{cap}\left(s^{*}, \mathscr{D}\right)+\operatorname{cap}\left(s^{*}, t\right) \\
& -\operatorname{odd}(Q) .
\end{aligned}
$$

We can substitute $\operatorname{cap}\left(s^{*}, t\right)=a(V)-\operatorname{def}(\mathcal{M})$, $\operatorname{cap}\left(\mathscr{B}^{\prime}, t\right)+\operatorname{cap}\left(\mathscr{B}^{\prime}, t^{*}\right)=b(\mathscr{B}), \operatorname{cap}\left(\mathscr{C}^{\prime}, t^{*}\right)=a(\mathscr{C})$, and $\operatorname{cap}\left(\mathscr{A}, \mathscr{A}^{\prime}\right)+\operatorname{cap}\left(\mathscr{A}, \mathscr{D}^{\prime}\right)+\operatorname{cap}\left(\mathscr{C}, \mathscr{A}^{\prime}\right)=c(\mathscr{A}, \mathscr{A} \cup \mathscr{C} \cup$ $\mathscr{D})$. Proceeding from the canonical decomposition to the canonical barrier, we obtain

$$
\begin{aligned}
\operatorname{balcap}(Q)= & c(Y, Y \cup Z)+b(X)+a(Z) \\
& +a(X)+a(V)-\operatorname{def}(\mathcal{M})-\operatorname{odd}(X, Y) .
\end{aligned}
$$

The value of a maximum balanced $s^{*} t^{*}$-flow on $N_{M}^{\#}$ is $2 a(V)--2 \operatorname{def}(\mathcal{M})$, which is a lower bound on balcap $(Q)$. Comparison with (21) yields inequality (18) when $k:=\operatorname{def}(\mathcal{M})$ is chosen. Moreover, equality in (18) is reached if $Q$ is canonical so that $\operatorname{def}(\mathcal{M})$ is the maximum value of $k$ which applies.

Adding $c(X, Y)-a(Y)$ to both sides in (18) yields inequality (19).

\section{CAPACITY SCALING ALGORITHMS}

We conclude the discussion by a simple application of the duality relationship between balanced flows and edge cuts:

The simple augmentation algorithms given in [3] perform poorly if the arc capacities grow large. The simplest way to make augmentation algorithms polynomial is called capacity scaling. Actually, there are two similar algorithms for the maximum balanced-flow problem which depend on this idea.

Let $U$ denote an upper bound on the arc capacities in a balanced-flow network $N$ (with $k=\lceil\log U\rceil$ the length of integer representation for instance). Instead of $N$, we can apply an augmentation algorithm to a series $N_{1}, N_{2}, \ldots, N_{k}$ of balanced-flow networks. These networks consist of the original digraph but have arc capacities $\operatorname{cap}_{i}(a):=\operatorname{cap}(a) \bmod 2^{i}$ for every $a \in A(N)$.

The respective maximum flows are not computed from scratch. Instead, the initial flow for the network $N_{i}$ is $f_{i}: \equiv 2 f_{i-1}$, where $f_{i-1}$ is the maximum flow for $N_{i-1}$, which has been computed before. We may choose $f_{0}: \equiv 0$ in the very beginning. It is obvious that the resulting procedure determines a maximum balanced flow on $N=N_{k}$. We merely have to consider the total effort.

Let $Q_{i}$ be a minimum balanced cut for $N_{i}$, that is, $\operatorname{val}\left(f_{i}\right)=\operatorname{balcap}\left(Q_{i}\right)$ at the end of the $(i)$-scaling phase. If we proceed from $N_{i}$ to the network $N_{i+1}$, then an arc $a$ with $\operatorname{rescap}_{i}(a)=0$ has $\operatorname{rescap}_{i+1}(a) \leq 1$ and an $\operatorname{arc} a$ with $\operatorname{rescap}_{i}(a)=1$ has $\operatorname{rescap}_{i+1}(a) \leq 3$. We obtain

$$
\operatorname{rescap}_{i+1}\left(Q_{i}\right) \leq\left|Q_{i}\right|+2 o d d_{i}\left(Q_{i}\right) \leq m+2 n \text {. }
$$

There are $O(m)$ augmentation steps during one scaling phase, each of which needs $O(m \alpha(m, n))$ time. Hence, the time complexity of this capacity scaling algorithm is $O\left(m^{2} \log U \alpha(m, n)\right)$.

We mention that every but the last scaling phase may run with a BNS heuristic (like the Kameda/Munro algorithm described in [3] or the Pape/Conradt algorithm which only finds strictly simple augmenting paths). Then, correctness is maintained while polynomial performance cannot be guaranteed any longer. 
Another capacity-scaling algorithm runs the BNS on a modified residual network $N(f, \Delta)$ which consists of all arcs $a$ with $\operatorname{rescap}(a) \geq \Delta$. The procedure is started with $\Delta:=2^{i}, i:=k-1$. Whenever no augmenting path can be found, we put $i:=i-1$ (until $i=0$ is reached).

If we would combine this algorithm with a BNS procedure, there would be no improvement compared with the former algorithm. Instead of this, we determine arbitrary augmenting paths $p$ unless $\Delta=1$ and augment on $p$ and $p^{\prime}$ simultaneously. Each step increases the flow value by at least $\Delta$ likewise.

At the end of the (i)-scaling phase, we have $\operatorname{rescap}(S, T)<\Delta m$ for some st-cut $[S, T]$. Hence, at most $2 m$ augmentation steps can occur during the $(i+1)$ scaling phase. One gets the bound $O\left(m^{2} \log U\right)$ as before.

It may turn out that this bound can be improved to $O(n m \log U)$ by an adaption of labeling techniques described for the usual max-flow problem and a corresponding SAP scaling algorithm (see Ahuja et al. [1], pp. 210-220). Our experience with the phase-ordered augmentation algorithms in [4] indicates, however, that unexpected difficulties may arise when max-flow techniques are applied to matching problems.

\section{REFERENCES}

[1] R. Ahuja, T. Magnanti, and J. Orlin, Network flows, Prentice Hall, Englewood Cliffs, NJ, 1993.

[2] C. Fremuth-Paeger and D. Jungnickel, Balanced network flows (I): A unifying framework for design and analysis of matching algorithms, Networks 33 (1999), 1-28.

[3] C. Fremuth-Paeger and D. Jungnickel, Balanced network flows (II): Simple augmentation algorithms, Networks 33 (1999), 29-41.

[4] C. Fremuth-Paeger and D. Jungnickel, Balanced network flows (III): Strongly polynomial augmentation algorithms, Networks 33 (1999), 43-56.

[5] D. Jungnickel. Graphs, networks and algorithms, Springer, Heidelberg, Germany, 1999.

[6] W. Kocay and D. Stone, Balanced network flows, Bull ICA 7 (1993), 17-32.

[7] L. Lovasz, Subgraphs with prescribed valencies, J Combin Theory 8 (1970), 391-416.

[8] L. Lovasz and M. Plummer, Matching theory, NorthHolland, Amsterdam, 1986.

[9] W. Tutte, The factors of graphs, Can J Math 4 (1952), 314 328.

[10] W. Tutte, Antisymmetrical digraphs, Can J Math 19 (1967), 1101-1117. 